\title{
Integrating primary and community care: an international perspective
}

In March 2018 the European Forum for Primary Care and the University of Kent in England, collaborated to bring together key international speakers in a Symposium to discuss approaches to integrating primary and community care across Europe, and to consider the achievements and challenges to date and the way forward for Primary Care in the future. This took place over one day, 2nd March 2018, in the city of Canterbury, England and coincided with some of coldest weather in south-east England on recent record.

The term integration has become a significant policy driver over recent years in relation to health care and health systems. What does it really mean and why does it matter?

According to the Nuffield Trust 'Integrated care is an organizing principle for care delivery that aims to improve patient care and experience through improved coordination. Integration is the combined set of methods, processes and models that seek to bring this about' (2011: p. 3).

This concept has been taken up and accepted in the context of primary health care over the last decade as exemplified in the World Health Organisation (WHO) (2008) report Primary Health Care Now More than Ever. In 2018 the European Commission Expert Panel opinion on Primary Care defined primary care:

\section{'The Expert Panel considers that primary care is the provision of universally accessible, integrated person-centered, comprehensive health and community services provided by a team of professionals accountable for addressing a large majority of personal health needs. These services are delivered in a sustained partnership with patients and infor- mal caregivers, in the context of family and community, and play a central role in the overall coordination and continuity of peo- ple's care' (EU Commission, 2018: p. 9).}

In most European countries the Primary Care system exists to provide family and patientcentered care, usually led by family or general practitioners with a supportive team of nurses and other health professionals, to enable people to manage their health and health care outside of hospital and to act as a gateway into the acute care hospital system or tertiary care as it becomes necessary. It is often (although not always) the first point of contact in the health care system. The funding model for Primary Care varies across Europe from per capita funding to GPs through taxation to private provision through insurance, sometimes with additional incentive and target related payments from the state.

In addition to Primary Care countries also operate other community-based services that are provided for the same patients and their families but frequently fall under different funding mechanisms, different organizational arrangements and different kinds of health care provision, and, critically, different information management systems. All these parts of the system can work separately under their own governance and finance arrangements to provide the care the patient needs but the care can be fragmented, un-coordinated and incur unnecessary delays and difficulties for the patient and the family, especially as un-coordinated information management systems can mean that none of the parts of the patient's whole care pathway have the complete picture. This can have a huge impact on those patients who are the most vulnerable and with very complex care needs. Bringing these fragments of primary and community care together provides a much more positive patient experience, improves outcomes and enables a more costefficient service to be provided (Rayner et al., 2018). In England the New Models of Care (Department of Health, 2015) policy has been implemented in the NHS to enable such a process of integration to be developed, piloted and evaluated. Other countries such as Belgium, Netherlands, Spain and Portugal have also made advances in integrating care in the community. The Symposium provided an opportunity to review some of the advances in integrating care, and the challenges, across different populations and systems. 
One of the greatest challenges of our time is the financing and management of Primary Care in a way that people are not put at risk of financial hardship at the point of service for all citizens, based on the Declaration of Alma Ata (WHO, 1978) that Primary Care should be accessible to all. In a world where there is economic austerity, demographic trends towards an older population living longer with more complex needs and a rise in chronic conditions such as diabetes, obesity and mental health problems, as well as poor nutrition, poverty, migration and the trauma of war, the concept of Universal Health Coverage (UHC; WHO, 2017) is a massive challenge to meet. And yet equity of access and care is a fundamental human right that $\mathrm{UHC}$ must be able to rise to especially in low- and middle-income countries. Professor Pavlos Theodorakis from the WHO Primary Care Centre, Almaty tackled this challenge head on as the opening speaker of the symposium. He provided an insightful overview of the background to the development of primary health care since the Declaration of Alma Ata in 1978, looking forward to 2030. Van Weel et al. (2008) have differentiated primary health care from Primary Care. proposing that primary care usually refers to personalized care whilst primary health care is a population based, community orientated approach to health care.

Recognizing the advances that many European states have made in strengthening Primary Care in recent years, his key message in the emerging health care context in Europe is that the lessons learned must be implemented to ensure universal coverage and equity of access. This means that, whilst recognizing the centrality of people centredness in primary care, countries and governments must pay attention to partnerships and relationships, population management, intersectoral care, evidence-based practice, outcomes, incentives, life course approaches, community orientation and, of course, integration of services to develop stronger primary health care.

Other key speakers provided research-based approaches as to how such integration and strengthening of Primary Care could enhance health outcomes for different populations. Professor Jenny Billings from University of Kent presented the work she has been leading on the Vanguard sites in England where the New Models of Care that were proposed in the NHS England strategy the Five Year Forward View (2015) are being tested. She highlighted the need for integration at systems, organizational, functional, professional and service levels in order to achieve both horizontal and vertical integration across the health care system. In her evaluation of a Multispeciality Community Provider in Kent she examined both the challenges of evaluation of complex organizational change and also the emerging outcomes of a fully integrated system. Notably there was a marked reduction in emergency admissions and in the number of hospital bed days used from the baseline, but equally service users have experienced a qualitative difference in their care. Billings identified key ingredients including strong GP leadership, adequate administrative services, supporting key workers and disseminating information in a timely way, IT infrastructure supporting communication, improving working relationships, managers affording the workforce time to participate, having regular multi-agency attendance at meetings, a shared understanding, trust and commitment, consistent and convenient meeting times. Such ingredients seem common sense in today's world but complexity of finance, procurement and delivery, increasing demand and supply, and inconsistency in political processes converge to make organizational decision making and implementation of policy increasingly challenging. Professor Henk Parmentier from the World Federation for Mental Health made a strong case for integration of primary care with other parts of the health system for people with mental illness, highlighting that many with a mental health problem such as major depression also have other long-term conditions such as diabetes and that people with long-term conditions often have an underlying mental health problem. By 2030 depression will be the commonest illness seen in primary care, the need to ensure that primary care teams are working together with acute, community and voluntary services is imperative for this particularly vulnerable population who may be living on the margins of society. Parmentier also remarked on the problems of culture how people both seek and use health care can make a huge difference to the way in which the system delivers care to the vulnerable.

Professor Jan de Maeseneer from University of Ghent extended the discussion of vulnerability and the need to consider how we think of primary 
health care as community-orientated and primary care as personalized care and bring these concepts together as an integrated system. Using the example of a community-orientated primary care centre in Ghent he demonstrated how a highly diverse community can be provided with a level of care that meets both individual expectations and goals of health care and the policy demands of a population based system. Again, this relies on similar indicators that Billings refers to - strong teams, leadership, communication, education, patient involvement and participation, intersectoral working - the key is the political will and organizational and systems know-how to enable change at both local and national levels.

Professor Kate O'Donnell from Glasgow University focused on particularly vulnerable groups and the problem of providing integrated health care to those who are migrant, the marginalized, refugees and asylum seekers from war torn and politically volatile countries who arrive in Europe in desperate need of health care. Issues of culture, racism, literacy and language are additional barriers for such people as well as the problem of older people's frailty and families with young children, infants and pregnant mothers.

In his concluding comments on the implementation of policies for integrated primary care systems, Professor Stephen Peckham from the University of Kent offered his analysis of the differing positions that policy implementation can take in a matrix of ambiguity and conflict (Matland, 1995). The matrix is derived from the national evaluation of the New Models of Care in England. In framing the lessons learned for scaling up primary care in other countries, Peckham concluded that a danger in policy implementation is that it can fall into the symbolic quadrant of the matrix where there is high ambiguity and high conflict, where there may be collaborative strength amongst professionals but a lack of clarity about what can be achieved and where neither top-down nor bottom-up stakeholders are committed to the process, leading to a disintegrated approach to implementation. For a more successful approach to scaling up integrated primary care, policy implementation is more likely to be effective where the degrees of conflict and ambiguity can be considered in relation to the context and the complexity of the policy and the appropriate measures adopted to ensure that implementation and delivery of integrated primary and community care policies and services can coalesce.

Despite a bitter easterly wind and snow, delegates from across Europe discussed these topics vigorously before leaving for their various destinations as far afield as Turkey and Brazil. Discussion will continue at the EFPC conference in Crete 24-25th September 2018 (http://www.euprimarycare.org/).

For further details of the presentations, please go to: https://www.kent.ac.uk/chss/events/EFPCmarch.html

\section{Sally Kendall \\ Professor of Community Nursing and Public Health, University of Kent, UK Editor in Chief Chair, European Forum for Primary Care}

\section{References}

Department of Health 2015: New models of care. London: DH. Matland, R.E. 1995: Synthesizing the implementation literature: the ambiguity-conflict model of policy implementation. Journal of Public Administration Research and Theory 5, 145-74.

NHS England 2014: The five year forward view. London: NHSE.

Rayner, J., Muldoon, L., Bayoumi, I., McMurchy, D., Mulligan, K. and Tharao, W. 2018: Delivering primary health care as envisioned: a model of health and well-being guiding community-governed primary care organizations. Journal of Integrated Care.

Van Weel, C., de Maeseneer, J. and Roberts, R. 2008: Integration of personal and community health care. The Lancet 372, 871-72.

World Health Organisation and World Bank 2017: Tracking Universal Health Coverage. Geneva: WHO.

World Health Organisation 2008: Primary Health Care Now More than Ever. Geneva: WHO. 